\title{
Increased incidence of biliary sludge and normal gall bladder contractility in patients with high spinal cord injury
}

\author{
R K Tandon, R K Jain, P K Garg
}

\begin{abstract}
Background-Patients with spinal cord injury (SCI) have an increased prevalence of gallstones.

Aims-To study prospectively the incidence of gallstones and gall bladder contractility in patients with SCI.

Patients and methods-Thirty six consecutive patients with SCI were studied: 18 patients with SCI above thoracic 10 neuronal segment (>T10) and 18 patients with SCI below T10 (<T10). An equal number each of disease controls (multiple fractures) and healthy controls were also studied. All patients and controls underwent serial ultrasonography to detect development of gallstones and ultrasonographic measurement of gall bladder contractility.

Results-A significantly higher number (9/18) of patients with SCI > T10 developed biliary sludge compared with patients with SCI <T10 (2/18), disease controls $(2 / 18)$, and healthy controls (1/18) $(p<0.05)$. No patient developed gallstones. The gall bladder fasting volume was significantly decreased in patients with SCI > T10 $(20.56 \mathrm{ml} ; 95 \%$ confidence intervals (CI) 19.74 to 21.38 ) compared with that in patients with SCI <T10 $(27.33 \mathrm{ml}$, 95\% CI 26.17 to 28.49 ; p<0.05), disease controls $(27.92 \mathrm{ml}, 95 \%$ CI 26.69 to 29.15 ; p<0.05), and healthy controls $(28.35 \mathrm{ml}$, $95 \%$ CI 27.25 to $29.45 ; p<0.05)$. Gall bladder contractility was normal in patients with SCI as shown by normal gall bladder residual volume and emptying time.
\end{abstract}

Conclusions-Patients with SCI above T10 have an increased incidence of biliary sludge and a decreased gall bladder fasting volume. Gall bladder contractility is, however, normal.

(Gut 1997; 41: 682-687)

Department of

Gastroenterology,

All India Institute of

Medical Sciences,

New Delhi 110 029,

India

R K Tandon

R K Jain

P K Garg

Correspondence to: Professor R K Tandon.

Accepted for publication 12 May 1997

Spinal cord injury (SCI) is a worldwide problem. ${ }^{12}$ Many gastrointestinal complications have been observed in patients with SCI. These include constipation, abdominal distension, altered gastrointestinal motility, and increased prevalence of gallstones. $^{34} \mathrm{~A}$ necropsy study and a prevalence study have shown the prevalence of gallstones in patients with SCI to be $29-49 \% .^{56}$ The possible reasons considered for increased prevalence of gallstones were altered gastrointestinal and gall bladder motility, decreased food intake, parenteral nutrition, and altered metabolic state with peripheral fat mobilisation secondary to prolonged confinement to bed..$^{57}$ The basis for altered gall bladder and gastrointestinal motility was thought to be impaired sympathetic nerve supply (SNS) following SCI while the parasympathetic supply via the vagus nerve remained intact. ${ }^{35-7}$

Besides indicating the possible association of gallstone disease with SCI, these studies also indicated that two important aspects of this association merited further investigation. Firstly, the evolution of gallstones following spinal cord injury is not known because patients with SCI have not been studied prospectively starting soon after injury. Secondly, even though the SNS to the gall bladder is known to originate from thoracic spinal segments $7-10^{8}$ and impaired SNS has been implicated as the probable cause for increased gallstone formation and altered gall bladder motility, whether there is any difference in gallstone prevalence and gall bladder motility between patients with SCI above thoracic 10 (T10) segment (impaired SNS) and patients with SCI below T10 (intact SNS) has not been studied.

We thus undertook the present study with a working hypothesis that gallstones might not be present with increased frequency in patients with SCI soon after injury but may possibly appear during follow up; and that there might be differences in the incidence of gallstones and gall bladder contractility in patients with SCI above T10 and those with SCI below T10.

\section{Materials and Methods}

All patients with SCI admitted to the orthopaedic ward of the All India Institute of Medical Sciences, New Delhi, a tertiary care centre, during the period July 1993 to June 1994, were included in the present study and followed up prospectively.
EXCLUSION CRITERIA

Patients with spinal cord diseases such as spinal tuberculosis and spinal cord tumours which cause incomplete and gradual spinal cord compression were not included. Patients with other risk factors for gallstones, for example, haemolytic disorders, vagotomy and gastric surgery, cirrhosis of the liver, ileal diseases, diabetes mellitus, obesity, and intake of drugs 
such as nicotinic acid, clofibrate, and oral contraceptives would also have been excluded, although no such patients were encountered. None of the patients were on regular nonsteroidal anti-inflammatory drugs. Patients with long standing (years) chronic spinal cord injury were not included.

\section{CONTROL GROUPS}

Two control groups were included. One group was comprised of normal healthy volunteers and the other group of patients with multiple fractures who were likely to be confined to bed for a minimum period of 12 weeks, for example, patients with pelvic fractures. The latter group of patients served as disease controls to see the effect of low physical activity (secondary to bed confinement) on the incidence of gallstone formation and on gall bladder contractility.

All study subjects underwent thorough history taking and detailed clinical examination. The level of spinal cord injury was determined by detailed neurological examination supported by radiological investigations. The degree of neurological damage was graded according to Frankel et al. ${ }^{9}$ Laboratory investigations performed on these patients included haematological and serum biochemical tests such as glucose, urea, bilirubin, alkaline phosphatase (AP), and alanine and aspartate aminotransferases (ALT and AST).

These patients were being treated by orthopaedic surgeons in the standard manner including spinal traction, if required. They were being fed orally. None of the study patients required parenteral nutrition. A dietitian was involved in the management of these patients and a diet containing at least 8.36$10.45 \mathrm{MJ} /$ day (approximately $60-70 \%$ as carbohydrate, $20-30 \%$ as fat, and $20 \%$ as proteins) was given to each patient through three regular meals with snacks in between. We could not measure the weight of our patients regularly because we do not have weighing beds for such patients. However, none of our patients lost any appreciable weight during the period of study due to a proper diet schedule.

\section{ULTRASONOGRAPHIC DETECTION OF}

GALLSTONES/BILIARY SLUDGE

Ultrasonographic examination of the abdomen was done in patients and controls at the bedside after an overnight fast within seven days after admission to the hospital and subsequently at $2,4,8,12,16$, and 24 weeks to determine the incidence of gallstones. The ultrasound machine used for the purpose was Medirad (General Electricals) with a $3.5 \mathrm{MHz}$ probe. The ultrasonologist was kept blind to the level of spinal cord injury in these patients. Biliary sludge was defined as echogenic material inside the gall bladder with layering and movement with change of posture but without acoustic shadowing.

MEASUREMENT OF GALL BLADDER CONTRACTILITY BY ULTRASONOGRAPHY Gall bladder contractility was measured in patients and controls at the same time as abdominal ultrasonography was being performed for detecting gallstones/sludge. Gall bladder contractility was measured only once at the start. Gall bladder volume was measured by utilising the ellipsoid formula-that is, volume $=0.52 \times$ (width $\times$ height $\times$ length $).{ }^{10}$ After recording fasting volume (= resting volume, FV) a standard meal was given containing approximately $1.672 \mathrm{MJ}$ (two slices of bread, $20 \mathrm{~g}$ butter, and $200 \mathrm{ml}$ milk). Gall bladder volume was measured after the meal at 5, 15, and every 15 minutes thereafter until such time as no further contraction of the gall bladder was observed or gall bladder volume increased over the previous value. Residual volume (RV) (smallest gall bladder volume observed at any time after ingestion of food) was then recorded. Emptying time (interval between the time from ingestion of first mouthful of meal and the time at which no further contraction of gall bladder occurred) and ejection fraction $(\mathrm{EF}=(1-\mathrm{RV} / \mathrm{FV}) \times 100)$ were then calculated. All measurements were made in duplicate and the average values were used for the calculation.

STATISTICAL ANALYSIS

Data are expressed as mean (SD) and 95\% confidence intervals (CI) are also given as appropriate. Statistical analysis was performed using one way analysis of variance and the multiple range test for comparison of continuous data among different groups. The $\chi^{2}$ test with Yates's correction was used for comparing categorical data between any two groups. The Mantel Cox test was used for comparison of non-parametric data. The Kaplan-Meier survival analysis method was used to depict cumulative probabilities of development of biliary sludge in patients and controls. A $p$ value of less than 0.05 was regarded as significant.

All study subjects were included after obtaining informed consent and the study protocol conformed to the ethical guidelines of the 1975 Declaration of Helsinki.

\section{Results}

There was a total of 37 patients with SCI. These patients were divided into two groups according to the level of injury: group I, SCI above T10 level (>T10, high SCI); and group II, SCI below T10 (<T10, low SCI). There were 19 patients in group I and 18 patients in group II. One patient in group I with a long standing SCI of six years duration was excluded. Thus, there were 18 patients in each group. In addition, 18 "disease" controls (group III) and 18 healthy controls (group IV) were studied.

DEMOGRAPHIC FEATURES AND SERUM

BIOCHEMISTRY

There was no statistically significant difference between any two groups with regard to demographic data, for example, age and sex ratio, and biochemical parameters (table 1).

LEVEL AND GRADE OF SCI

Table 2 shows levels and grades of SCI. The majority of patients in group I had cervical 
TABLE 1 Demographic features and biochemical parameters of patients and controls

\begin{tabular}{lllll}
\hline Mean & Group I & Group II & Group III & Group IV \\
\hline Age (years) & $31.5(13.2)$ & $31.6(12.1)$ & $28.3(13.3)$ & $31.75(13.32)$ \\
Sex ratio (M:F) & $14: 4$ & $12: 6$ & $15: 3$ & $13: 5$ \\
Level of SCI & $>$ T10 & $<$ T10 & NA & NA \\
$\begin{array}{l}\text { Mean duration of injury } \\
\quad(\text { days) }\end{array}$ & 36.9 & 15.3 & 53.4 & NA \\
Quadriplegia & $15 / 18$ & $0 / 18$ & NA & NA \\
Paraplegia & $3 / 18$ & $18 / 18$ & NA & NA \\
Haemoglobin (g/dl) & $10.58(1.72)$ & $10.95(1.73)$ & $10.11(2.93)$ & $10.54(1.62)$ \\
Blood glucose (mg/dl) & $104.77(22.02)$ & $97.11(20.47)$ & $98.42(20.34)$ & $90.85(16.00)$ \\
Blood urea (mg/dl) & $23.77(6.57)$ & $29.27(11.89)$ & $29.05(13.08)$ & $27.55(8.03)$ \\
Bilirubin (mg/dl) & $0.91(0.24)$ & $0.91(0.19)$ & $0.98(0.17)$ & $0.85(0.29)$ \\
AP (KA) & $10.05(1.79)$ & $10.05(1.95)$ & $9.31(2.74)$ & $10.15(2.75)$ \\
AST (IU) & $34.22(10.79)$ & $42.11(23.26)$ & $33.68(13.56)$ & $30.70(10.28)$ \\
ALT (IU) & $31.61(11.80)$ & $39.88(31.37)$ & $32.26(11.76)$ & $27.45(7.62)$ \\
Cholesterol (mg/dl) & $205.88(31.51)$ & $211.22(23.39)$ & $202.94(22.02)$ & $194.05(25.46)$ \\
\hline
\end{tabular}

TABLE 2 Level and grade of SCI in group I and group II patients

\begin{tabular}{lll}
\hline & Group I (n) & Group II (n) \\
\hline Level of SCI & 2 & 0 \\
C2-3 & 8 & 0 \\
C4-5 & 6 & 0 \\
C6-7 & 1 & 0 \\
T1-5 & 1 & 0 \\
T6-10 & 0 & 11 \\
T11-12 & 0 & 7 \\
L1-5 & 10 & 12 \\
Grade of SCI & 8 & 6 \\
Frankel grade A & Frankel grade B &
\end{tabular}

C, cervical spinal cord; $T$, thoracic spinal cord; $L$, lumber spinal cord.

involvement. All patients had complete or near complete SCI - that is, Frankel's grade A or B.

DURATION OF INJURY

The duration of SCI before hospitalisation varied from 1 to 180 days with a median of 30 days in group I and was 2-75 days with a median of 11 days in group II. There was no statistical difference between these two groups with regard to the duration of injury. Patients were studied within seven days after admission to the hospital.

The mean follow up of patients in group I was 10.4 weeks (range 8-24 weeks), in group II 8.6 weeks (range $4-20$ weeks), and in group III 8.8 weeks (range $8-24$ weeks).

\section{GALL BLADDER CONTRACTILITY}

Table 3 presents the results of various gall bladder contractility tests.

\section{Gall bladder fasting volume}

The gall bladder fasting volume in group I (20.56 ml, $95 \%$ CI 19.74 to 21.38 ) was significantly less than that in group II $(27.33 \mathrm{ml}, 95 \%$ CI 26.17 to $28.49 ; \mathrm{p}<0.05)$, group III (27.92 $\mathrm{ml}, 95 \%$ CI 26.69 to 29.15 ; $\mathrm{p}<0.05)$,

TABLE 3 Gall bladder volumes, ejection fraction, and emptying time in patients and controls

\begin{tabular}{|c|c|c|c|c|}
\hline & Group I & Group II & Group III & Group IV \\
\hline $\mathrm{GBFV}^{\star}(\mathrm{ml})$ & $20.56(0.43)$ & $27.33(0.59)$ & $27.92(0.63)$ & $28.35(0.56)$ \\
\hline GBRV (ml) & $10.21(0.26)$ & $11.31(0.31)$ & $10.76(0.31)$ & $10.27(0.52)$ \\
\hline $\mathrm{GBDV}^{\star}(\mathrm{ml})$ & $10.34(0.21)$ & $16.02(0.39)$ & $17.22(0.43)$ & $18.08(0.53)$ \\
\hline $\mathrm{EF}^{\star}(\%)$ & $50.48(0.51)$ & $58.57(0.70)$ & $61.02(0.54)$ & $63.12(1.50)$ \\
\hline Emptying time ( $\mathrm{min})$ & $43.33(0.57)$ & $42.77(0.60)$ & $41.66(0.57)$ & $45.83(1.41)$ \\
\hline
\end{tabular}

GBFV, gall bladder fasting volume; GBRV, gall bladder residual volume; GBDV, gall bladder delta volume; EF, ejection fraction. Results expressed as mean (SEM).

${ }^{\star}$ Group I $v$ group II, $\mathrm{p}<0.05$; group I $v$ group III, $\mathrm{p}<0.05$; group I $v$ group IV, $\mathrm{p}<0.05$. and group IV $(28.35 \mathrm{ml}, 95 \%$ CI 27.25 to $29.45 ; \mathrm{p}<0.05)$. There were no differences between groups II and III, II and IV, and III and IV.

\section{Gall bladder residual volume}

There was no significant difference in the gall bladder residual volume among all four groups.

\section{Gall bladder delta volume}

The delta volume-that is, the difference between the fasting volume and the residual volume, was significantly lower in group I $(10.34 \mathrm{ml}, 95 \%$ CI 9.92 to $10.76 ; \mathrm{p}<0.05)$ than delta volumes in group II $(16.02 \mathrm{ml}, 95 \%$ CI 15.24 to $16.80 ; \mathrm{p}<0.05)$, group III (17.22 ml, 95\% CI 16.36 to $18.08 ; \mathrm{p}<0.05)$, and group IV $(18.08 \mathrm{ml}, 95 \%$ CI 17.02 to 19.14; $\mathrm{p}<0.05)$.

\section{Ejection fraction}

Gall bladder ejection fraction in group I (50.48; $95 \%$ CI 49.48 to 51.48$)$ was significantly less than that in group II $(58.57,95 \%$ CI 57.20 to $59.94 ; \mathrm{p}<0.05)$, group III (61.02, $95 \%$ CI 59.97 to $62.07 ; \mathrm{p}<0.05)$, and group IV $(63.12,95 \%$ CI 60.19 to $66.05 ; \mathrm{p}<0.05)$. It was, however, not found to be different between groups II and III, II and IV, and III and IV.

\section{Emptying time}

There was no statistical difference in gall bladder emptying time among all four groups.

\section{INCIDENCE OF BILIARY SLUDGE}

Biliary sludge was found in two patients in group I at the time of entry into the study and developed subsequently in an additional seven patients in the same group during follow up (table 4). The duration of SCI in the two patients who had biliary sludge at entry into the study was 30 and 35 days, respectively. Biliary sludge persisted in all nine patients throughout the period of study. There was no suggestion that patients with higher lesions had more chances of developing biliary sludge.

One group II patient had biliary sludge at entry into the study and one more patient developed sludge during follow up after two weeks. In group III one patient had biliary sludge at onset and another developed biliary sludge during follow up. One healthy control also was found to have biliary sludge.

There were statistically significant differences between group I and group II ( $p<0.05$; odds ratio 8), group I and group III ( $\mathrm{p}<0.05$; odds ratio 16), and group I and group IV $(\mathrm{p}<0.05$; odds ratio 16$)$ with regard to incidence of biliary sludge. Figure 1 shows the cumulative probabilities of developing biliary sludge in patients and controls.

INCIDENCE OF GALLSTONES

None of the patients in any group were found to have gallstones at entry into the study and none developed gallstones subsequently during follow up. 
TABLE 4 Development of biliary sludge

\begin{tabular}{ll}
\hline & Biliary sludge \\
\hline Group I & $9 / 18^{\star}(50 \%)$ \\
C2-3 & $1 / 2$ \\
C4-5 & $4 / 8$ \\
C6-7 & $3 / 6$ \\
T1-5 & $0 / 1$ \\
T6-10 & $1 / 1$ \\
Group II & $2 / 18(11 \%)$ \\
Group III & $2 / 18(11 \%)$ \\
Group IV & $1 / 18(6 \%)$ \\
\hline
\end{tabular}

${ }^{\star} \mathrm{p}<0.05 v$ group II, $v$ group III, and $v$ group IV.

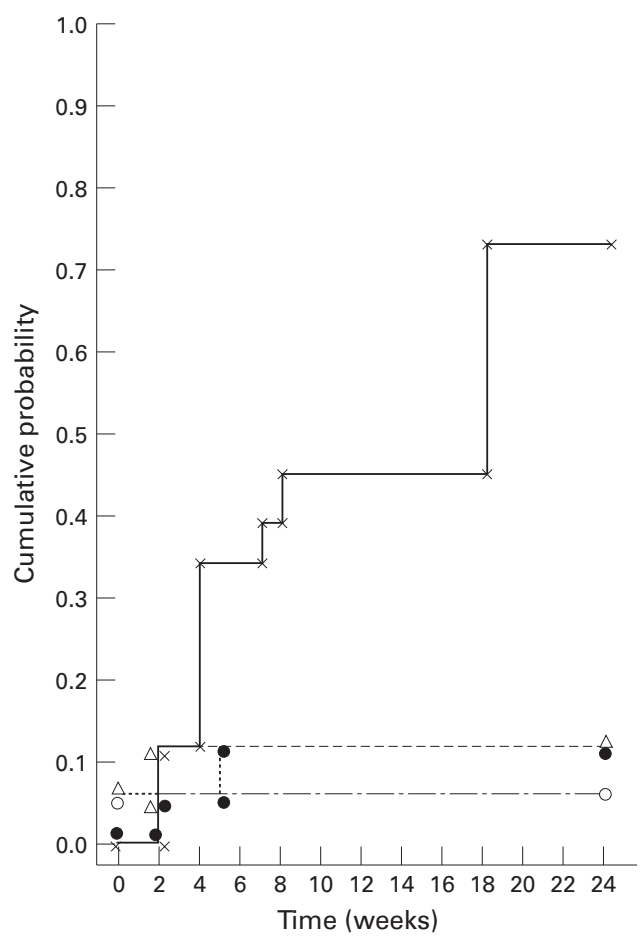

Figure 1: The cumulative probabilities of developing biliary sludge with regard to the time of injury (taken as time 0).Crosses represent SCI above T10, open triangles SCI below T10, closed circles disease controls, and open circles healthy controls.

\section{Discussion}

Some interesting observations have emerged from our study. The most impressive and original of them is the increased incidence of biliary sludge only in patients with SCI above T10 and not in those with SCI below T10. This finding has not been reported previously. We included patients with neurologically complete or near complete SCI to preclude any effect of neurological instability in the early post-SCI phase. There was, in contrast to earlier studies, ${ }^{57}$ a substantial number of patients with SCI below T10 $(n=18)$ in our study; this was a major handicap in appreciating the role of disruption of the SNS on gall bladder contractility and gallstone formation in the earlier studies.

The present study also sheds light on gall bladder physiology related to contractility. To assess gall bladder contractility, various parameters have been recommended such as gall bladder fasting volume, residual volume, delta volume, ejected volume, emptying time, and ejection fraction. In our study, the gall bladder fasting volume was found to be significantly decreased in patients with SCI above T10 compared with that in those with SCI below $\mathrm{T} 10$ and controls. A lower fasting volume indicates impaired gall bladder filling. Ejection fraction was also found to be significantly less in patients with SCI above T10 as compared with that in patients with SCI below T10 and controls. However, there was no difference in the gall bladder residual volume or emptying time between any two of the four groups of patients studied. A decrease in ejection fraction in the face of no decrease in gall bladder residual volume suggests that the observed decrease in ejection fraction was most likely due to decreased gall bladder fasting volume in these patients.

The delta volume ${ }^{11}$ - the difference between the gall bladder fasting volume and gall bladder residual volume - was low in patients with SCI above T10. This also was because of lower gall bladder fasting volume in these patients. Delta volume alone does not carry much significance and is not equivalent to the ejected volume. It has been shown that gall bladder filling continues during its contraction. ${ }^{11-13}$ The ejected volume of bile following a stimulus is the sum of the volume of bile already present in the gall bladder and a much larger volume of hepatic bile that enters the gall bladder during its contraction. Although we did not measure ejected volume in our patients because of methodological problems, we believe that due to the impaired gall bladder filling in these patients, the estimated ejected volume would be lower.

The obvious reason for lower fasting volume and ejection fraction in patients with SCI above T10 and not in patients with SCI below T10 could be a deranged sympathetic nerve supply to the gall bladder which is normally derived from T7-10 segments of the spinal cord. ${ }^{8}$ The decreased fasting volume in patients with SCI above T10 (impaired SNS) suggests that the SNS is probably responsible for gall bladder relaxation during its filling, which is in agreement with earlier observations. ${ }^{14-16}$

Thus, except for impaired gall bladder relaxation leading to a lower fasting volume in patients with SCI above T10, gall bladder contractility was found to be normal in patients with SCI. Only one other study is available on sonographic measurement of gall bladder contractility in patients with SCI which has shown almost similar findings. ${ }^{7}$ However, there were only four patients with SCI below T10 in that study which precluded a meaningful comparison between patients with SCI above T10 and those with SCI below T10. Two studies on scintigraphic measurement of gall bladder contractility (published as abstracts ${ }^{17}{ }^{18}$ ) showed that gall bladder contractility was abnormal in patients with SCI. The differences in the results of these earlier studies may possibly be explained on the basis of differences in methods (sonographic versus scintigraphic) and in patient populations with regard to the level, duration, and completeness of spinal cord injury. ${ }^{77-22}$ However, since cholecystokinin and vagal responses are preserved in patients with SCI and only the SNS to the gall bladder is impaired, our findings of normal gall bladder 
contractility and lower gall bladder fasting volume (suggestive of impaired gall bladder filling) in patients with SCI above T10 conform well to the established physiological facts about gall bladder contractility - that is, cholecystokinin and the vagus are important for gall bladder contraction, ${ }^{14}$ whereas the sympathetic nerve supply is mainly responsible for gall bladder relaxation during filling. ${ }^{14} 16$

The cause of biliary sludge formation in patients with spinal cord injury remains to be determined. None of the known predisposing factors for the development of biliary sludge such as prolonged fasting, parenteral nutrition, rapid weight loss, pregnancy, etc, ${ }^{23}$ were present in our patients with SCI. The noted difference between patients with SCI above T10 and SCI below T10 with regard to biliary sludge formation gives credence to our hypothesis that interference with the SNS to the gall bladder may have an important bearing on the development of biliary sludge. It has been shown that gall bladder stasis may promote sludge formation. ${ }^{24}$ We could not however, find any suggestion of gall bladder stasis in our patients who developed sludge; the only abnormality found during the study of gall bladder contractility was decreased gall bladder fasting volume with a consequent decrease in gall bladder ejection fraction. Although it seems that only this alteration in gall bladder physiology may not be an adequate explanation for sludge formation, a lower fasting gall bladder volume may play an as yet undefined role in the pathogenesis of biliary sludge.

It is possible that the SNS could promote sludge formation through its inhibitory effect on intestinal motility following SCI. ${ }^{34}$ The resultant delay in gut transit would lead to a relative increase in deoxycholic acid in the bile acid pool, which may result in lithogenic bile. ${ }^{25}$ We could not however, measure intestinal transit in our patients to test this hypothesis.

Acute trauma has been shown to be a risk factor for biliary sludge. ${ }^{26}$ Although we did not measure peripheral fat mobilisation in our patients, any significant effect of low physical activity with its consequent metabolic changes including peripheral fat mobilisation in the pathogenesis of biliary sludge in patients with SCI was negated by the fact that the disease controls (with multiple fractures) with prolonged confinement to bed did not show any increased tendency for sludge formation.

None of the patients in our study was found to have gallstones. This is in contrast to earlier reports which showed an increased prevalence of gallstones in patients with SCI. ${ }^{5}{ }^{6}$ There are however certain important differences between this and other studies, particularly with regard to selection of patients and methodology. One of the earlier studies, ${ }^{5}$ was based on autopsy findings and another was a prevalence study. ${ }^{6}$ Both the earlier studies were performed on patients with long standing SCI. In contrast, we studied patients prospectively soon after they sustained SCI because we wanted to study the events occurring in relation to the gall bladder in the early post-SCI period. The median interval from the time of sustaining
SCI to inclusion in our study was 30 days in patients with high spinal cord injury (above T10) and 11 days in those with low spinal cord injury (below T10). Biliary sludge which may predispose to the development of gallstones ${ }^{20}$ was found in nine patients with SCI above T10. The mean follow up of these patients was 10.4 weeks (range 8-24 weeks), which was the average period of stay in the hospital for patients with spinal cord injury. We could not follow up these patients for long periods of time because of practical difficulties of restricted mobility of these patients. If followed for a longer period of time, gallstones might have developed in some of our patients. In fact, biliary sludge might be an intermediate step between spinal cord injury and development of gallstones.

We thus conclude that there is an increased incidence of biliary sludge in patients with high SCI (above T10). The gall bladder fasting volume and ejection fraction were significantly decreased in patients with SCI above T10, possibly due to a deranged sympathetic nerve supply which is responsible for relaxation of gall bladder during filling. The exact pathogenesis of biliary sludge remains to be established.

We are grateful to Professor P K Dave, Head of the Department of Orthopaedics, All India Institute of Medical Sciences, New Delhi, for permitting us to study patients under his care. This paper was presented in part at the Digestive Diseases Week, paper was presented in part at the Digestive Diseases We
1995, and abstracted in Gastroenterology (1995; 108: A438).

1 Dave PK, Jayaswal A, Kotwal PP, Biyani A, Bhattarcharye T. Clinico-epidemiological study of spinal cord injury patients. Indian fournal of Orthopedics 1994; 28: 39-45.

2 Anderson DW, McLaurin R. The national head and spinal cord injury survey. $\mathcal{F}$ Neurosurg $1980 ; 53: 51-8$.

3 Fealey RD, Szurszewski JH, Merritt JL, DiMagno EP. Effect of traumatic spinal cord transection on human upper gastro-intestinal motility and gastric emptying. Gastroenterology 1984; 87: 69-75.

4 Glick ME, MeshKinpour H, Haldeman S, Hoehler F, Downey N, Bradley WE. Colonic dysfunction in patients with thoracic spinal cord injury. Gastroenterology 1984; 86: 287-94.

5 Apstein MD, Dalecki-chipper-field K. Spinal cord injury is a risk factor for gallstone disease. Gastroenterology 1987; 92: 966-8.

6 Everson GT. Gall bladder function in gallstone disease. Gastroenterol Clin North Am 1991; 20: 84-110.

7 Nino-Murcia M, Burton D, Chang P, Stone J, Perkash I. Gall bladder contractility in patients with spinal cord injury: a sonographic investigation. AfR Am $\mathcal{F}$ Roentgenol 1990; 154: 521-4

8 Hogan WJ, Dodds WJ, Geenen JE. The biliary tract. In: Christensen J, Wingate DL, eds. A guide to gastrointestinal motility. Boston: Wright PSG, 1983: 157-97.

9 Frankel HL, Hancock C, Hyslop JH, Michaelis LS, Unger GH, Verman JDS, Walsh JJ. The value of postural reduction in the initial management of closed injuries of the spine with paraplegia and tetraplegia. Part I. Paraplegia 1962; 3: 179-92.

10 Dodds WJ, Groh WJ, Darweesh RM, Lawson TL, Kishk SM, Kern MK. Sonographic measurement of gall bladder volume. AfR Am f Roentgenol 1985; 146: 1009-11.

11 Howard PJ, Murphy GH, Dowling RH. Gallbladder emptying patterns in response to a normal meal in healthy subjects and patients with gallstones: ultrasound study. Gut subjects and patients

12 Lanzini A, Jazrawi RP, Northfield TC. Simultaneous quantitative measurements of absolute gallbladder storage and emptying during fasting and eating in humans. Gastroenterology 1987; 92: 852-61.

13 Jazrawi RP, Pazzi P, Petroni ML, Prandini N, Paul C, Adam JA, et al. Postprandial gallbladder motor function: refilling and turnover of bile in health and in cholelithiasis. Gastroenterology 1995; 109: 582-91.

14 Banfield WJ. Physiology of the gallbladder. Gastroenterology 1975; 69: 770-7.

15 Mack AJ, Todd JK. A study of human gall bladder muscle in vitro. Gut 1968; 9: 546-9.

16 Persson CGA. Dual effects on the sphincter of Oddi and gall bladder induced by stimulation of the right splanchnic nerves. Acta Physiol Scand 1973; 87: 334-43.

17 Williams W, Apstein M, Chassen S, Hackett M, Tow DE Gall bladder motility in spinal cord injury patients [abstract]. F Nucl Med 1987; 28: P596. 
18 Milne N, Segal JL, Rypins EB, Brunneman SR, Lyons KP Biliary kinetics in spinal cord injury [abstract]. F Nucl Med

19 Segal JL, Milne N. Gall bladder function in patients with spinal cord injury [letter]. AfR Am f Roentgenol 1991; 157: 412

20 Apstein MD, Kaplan S, Milne N. Gallbladder contractility

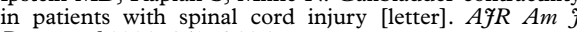
Roentgenol 1992; 158: 456-7.

21 Nino-Murcia N, Perkash I, Chang P. Reply to: Segal JL, Milne N. Gall bladder function in patients with spinal cord injury [letter]. AfR Am f Roentgenol 1991; 157: 412-3.

22 Nino-Murcia N, Perkash I, Chang P. Reply to: Apstein MD, Kaplan S, Milne N. Gallbladder contractility in patients with spinal cord injury [letter]. AfR Am f Roentgenol 1992; 158: 457.

23 Lee SP, Maher K, Nicholls JF. Origin and fate of biliary sludge. Gastroenterology 1988; 94: 170-6.

24 Roslyn JJ, Pitt HA, Mann LL, Ament ME, DenBesten L. Gall bladder disease in patients on long term parenteral nutrition Gastroenterology 1983; 84: 148-54.

25 Marcus SN, Heaton KW. Intestinal transit deoxycholic acid and the cholesterol saturation of bile-three inter-related factors. Gut 1986; 27: 550-8.

26 Imhof M, Raunest J, Ohmann C, Roher HD. Acute acalculous cholecystitis complicating trauma: a prospective study. World F Surg 1992; 16: 1160-6. 